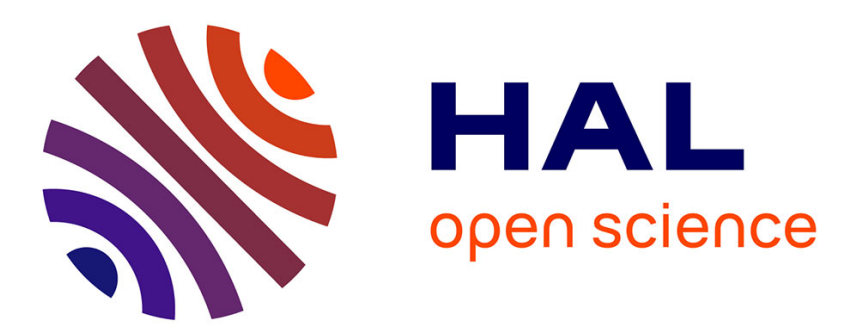

\title{
Cloud-connected flying edge computing for smart agriculture
}

\author{
M. Ammad Uddin, Muhammad Ayaz, Ali Mansour, El-Hadi Aggoune, Zubair \\ Sharif, Imran Razzak
}

\section{- To cite this version:}

M. Ammad Uddin, Muhammad Ayaz, Ali Mansour, El-Hadi Aggoune, Zubair Sharif, et al.. Cloudconnected flying edge computing for smart agriculture. Peer-to-Peer Networking and Applications, 2021, 10.1007/s12083-021-01191-6 . hal-03271416

\section{HAL Id: hal-03271416 \\ https://hal-ensta-bretagne.archives-ouvertes.fr/hal-03271416}

Submitted on 29 Jun 2021

HAL is a multi-disciplinary open access archive for the deposit and dissemination of scientific research documents, whether they are published or not. The documents may come from teaching and research institutions in France or abroad, or from public or private research centers.
L'archive ouverte pluridisciplinaire HAL, est destinée au dépôt et à la diffusion de documents scientifiques de niveau recherche, publiés ou non, émanant des établissements d'enseignement et de recherche français ou étrangers, des laboratoires publics ou privés. 


\title{
Cloud-Connected Flying Edge Computing for Smart Agriculture
}

\author{
M. Ammad Uddin ${ }^{1,2}$, Muhammad Ayaz ${ }^{1,2}$, Ali Mansour ${ }^{2}$, el-Hadi M. Aggoune ${ }^{1}$, Zubair Sharif ${ }^{3}$, Imran \\ Razzak $^{4}$ \\ ${ }^{1}$ Sensor Networks and Cellular Systems Research Center, University of Tabuk \\ 2 Lab STICC, ENSTA Bretagne, Brest, France \\ ${ }^{3}$ Department of CIS, Universiti Teknologi PETRONAS, Perak, Malaysia \\ ${ }^{4}$ Deakin University, Geelong, Victoria, Australia \\ Correspondence: mohammad.ammad-uddin@ensta-bretagne.org
}

\begin{abstract}
Due to recent advancements and the success of versatile mobile applications, more and more services around the globe are being moved to the cloud. As a result, its limitations have become more evident. The major issues that cloud-based applications face include large latency, bottlenecks because of central processing, compromised security, and lack of offline processing. The drawbacks of cloud computing are reduced by fog and edge computing, where data are processed near the places where it is generated-at network edges or fog nodes-most importantly in a distributed way. Smart agriculture is an approach based on the Internet of Things (IoT) where cloud computing is not an option as the internet is usually not available at remote sites. In addition, pure edge computing also is not practical, as most sensor nodes are very small and they do not have enough computing power. Intermediate fog computing also is not a good choice, as fixed fog nodes (getaway nodes) do not work well with high node fluctuation caused by bad weather or harsh conditions. Considering these issues and limitations, we have proposed the idea of flying edge computing where an unmanned aerial vehicle (UAV) acts as an edge-computing machine. This can be an ideal solution for smart agriculture, given the size and remoteness of many agricultural areas. This technique can be called "wind or breeze computing" because the data are "blown" or moved by the current of computing. The Flying-Edge offers fast deployment of edge facilities in challenging locations and it can be a major step to accomplish the goal of loT-based smart agriculture.
\end{abstract}

Keywords: Smart agriculture, Cloud computing, Edge computing, unmanned aerial vehicles, Security Issues

\section{Introduction}

The agriculture sector faces continuous pressure to feed the population of over 9 billion that is expected by 2050 amid challenges like climate change and the limited availability of arable land. To address this situation, scientists and researchers have suggested augmenting this sector with modern technologies including the Internet of Things (IoT) and unmanned aerial vehicles (UAVs) [1]. For example, loTs provide updates about crop health, which helps farmers make the right decision at the right time. Precision agriculture is all about collecting the right data at the right time so that the use of resources can be optimized by considering the requirements of every inch of farmland. To make agriculture sustainable using the loT has moved to the forefront of various agricultural operations. Moreover, UAVs are being used for various purposes in agriculture like monitoring, aerial sensing, tracking, data collection, communication, and security.

UAVs, more precisely called flying-robots, are used in a wide range of applications where they often act as the first responder because of their fast, cost-effective, and safe deployment capabilities [2][3][4]. Further, with improved technology and facilities like three-dimensional maneuverability, UAVs are playing the crucial role of airborne base stations in almost all emerging networks. Because 
of their involvement in a wide range of daily applications, UAVs have become the best candidates to achieve the goal of precision farming [5]. Due to these capabilities, UAVs can be used throughout the life cycle of crops in such functions as soil health inspections [6], plantation [7], plant counting [8], crop health monitoring [9] [10], irrigation, spraying fertilizers and pesticides [11], and estimating crop yield. This technology has become the point of interest for all the stakeholders related to agriculture-including farmers, agronomists, crop insurers, and researchers-because of its suitability and advanced functionalities. These include the variety of models, longer flight times, greater payload capacities, and the ability to fly in varying conditions. In this article UAVs are proposed as an option to overcome the wide range of challenges from remote agriculture:

- They need to cover large geographical areas.

- A wide range of sensors and other devices are needed to monitor various parameters in this highly heterogeneous environment.

- They need to operate in remote locations, where sensors operate unattended.

- Connecting the sensors is a challenge because of unreliable and fluctuating communications.

- Some parameters must report in real-time.

- Many parameters require scalable data storage and processing capabilities.

In this article, UAVs provide communication and data collection service to loT nodes. Once data are collected, the next question is where to process it and what computation model is best suitable to provide the required services to the loT nodes. For instance, cloud services can be used to access information from predictive analysis institutes so farmers apply products according to their specific requirements. However, for critical loT-based agriculture applications to operate in such challenging areas, they need a reliable communication medium between the deployed objects and processing devices, which cannot be guaranteed in large and remote areas.

Under these circumstances, cloud computing is not a good option because the internet may not be available in wide agriculture fields[12]. Furthermore, so-called fog computing also does not fit well since fog nodes are difficult to fix in harsh environments where node fluctuations are often. Most importantly, when we talk about specific applications in agriculture, edge computing may suffer from the fact that most nodes are very small, and they might not have sufficient processing capabilities. The need for scalability, economy, and node updates can generate more challenges. In short, in large farming areas where no reliable communication network is available to connect a large number of sensors to provide timely data, it is difficult to use existing fog and edge computing platforms.

Considering the challenge, this paper presents the idea of a Flying-Edge, a UAV-based computing platform that aims to fulfill the requirements of smart agriculture. Flying Edge platforms, which can comprise one or multiple UAVs, offer the computing capabilities of IoT applications operating in remote locations. The proposed platform is highly dynamic and flexible, so it can be deployed and can collect data quickly. Most importantly, it can be used in tough terrains like the desert and hilly areas, removing the barriers to smart agriculture in such locations. The proposed edge platform builds an loT network to collect crop data that will then be used to analyze crop health so, ultimately, the farmer can take timely smart actions.

The rest of the paper is organized as follows. Section 2 includes a short background of mobile edge computing. Section 3 provides the details of the proposed platform along with all the design and functionalities it can perform. Section 4 evaluates the proposed idea and compares the results of different parameters while, Section 5 presents concluding remarks and thoughts for future study. 


\section{Related Work}

Using mobile edge computing is not new. Various authors have proposed mobile systems that offer computing facilities. Prominent efforts include mobile fog computing [13] [14], mobile cloud computing [15], and mobile edge computing [16]. However, these platforms work differently from the proposed idea of flying edge computing. The system proposed in [13] offers mobile edge services from a fixed machine. It enables integrating mobile devices such as smartphones and vehicles to the cloud, but only from a certain geographical area. The important thing about that work is that it offers low-latency with the facility of mobility and location awareness. Further, [15] [17]proposes mobile cloud computing that offers an infrastructure where both data storage and data processing occur in the cloud to support mobile devices. In that approach, mobile cloud computing frees mobile devices from storing large data and conducting heavy processing. In [13], mobile edge computing extends cloud computing capabilities at the edge of the cellular network. Different services with low latency can be provided to smartphones and other applications that rely on cellular communications.

Using UAVs in smart agriculture also is not new. These flying robots are used in many ways. Authors in [18] present a hierarchical structure based on the collaboration between the UAVs and associated wireless sensors for crop monitoring in precision agriculture. That integration of UAVs with ground sensors and IoT applications proved to be a robust and efficient solution for data collection, control, analysis, and decisions in such specialized applications. Key advantages lay in online data collection and relaying the data to a central monitoring point, while effectively managing network load and latency through optimized UAV trajectories and in situ data processing. Two important aspects of the collaboration were considered: designing the UAV trajectories for efficient data collection and implementing effective data processing algorithms (consensus and symbolic aggregate approximation) at the network level to transmit the relevant data.

Considering the worth of Information and Communications Technology (ICT) for smart agriculture, researchers have begun to use edge computing to enhance crop production. In a similar effort in [19], the authors proposed a data link management solution based on mobile edge computing (MEC) to monitor water requirements for agriculture. The results showed that the scheme realizes the network and intelligence of agricultural water monitoring system management, and it has a broad market prospect.

Edge computing plays an important role in smart agriculture, and it can be considered an ideal paradigm because of features like its ability to operate in remote locations, cover large areas, endure hostile conditions, provide sufficient bandwidth, and address connectivity issues. Collecting data at the right time and then performing the right steps for a given situation is possible only by using edge computing. So, it is crucial for agriculture. Edge computing can be the appropriate choice whenever there are connectivity issues. According to the proposal in [20], sensors and edge devices collect data from the monitoring area and send the data to an edge node. The node conducts a preprocess analysis of the data needed then sends the outcome to a server. The server collects and accumulates the results to predict and estimate the total harvest yield. By using this framework many decisions can be taken according to circumstances. Authors in [21] focus on an efficient solution for collecting data and exploiting tasks using edge computing for smart agriculture, global food security, and the pervasive issue of internet access. Further, [22] presents a platform that can manage the minerals for agriculture using saline water. It relies on transferable low-cost hardware which operates with edge computing to interact with crop sensors and devices to collect the data and perform atomic control actions in real-time monitoring scenarios.

In all these studies, various features and services are provided to mobile devices to integrate with the cloud. However, they rely on fixed locations, such as stationary fog machines, fixed wireless 
stations, or fixed cellular towers. By contrast, Flying Edge offers these facilities from a mobile device instead of using only stationary devices.

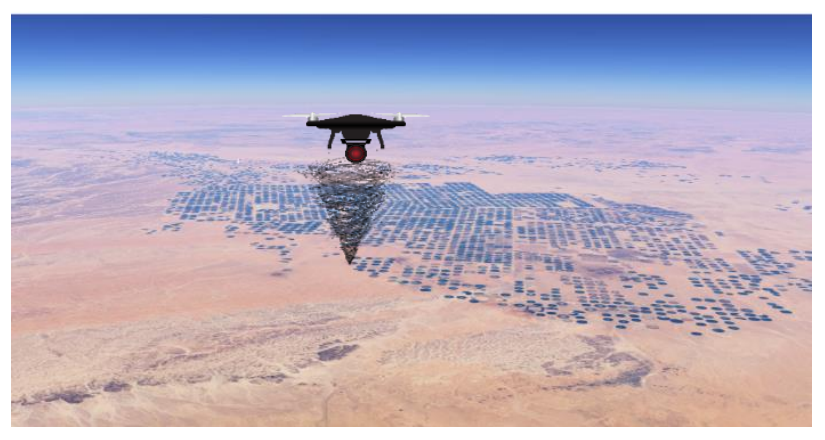

Figure 1: Tabuk region KSA agriculture farms and proposed flvina edae combutina

\section{Proposed Flying Edge Computing}

The traditional model has been to use cloud computing to acquire and process data from the edge to offer useful services. Large cloud data centers provide different services (computing, platform, security, and storage) to edge devices through high bandwidth connectivity. Two key challenges emerge with this hub-and-spoke model. Public cloud data centers are globally distributed, but they are typically limited to one or a few per country (depending on its size). The network distance from the edge to the cloud can result in a long round trip time (RTT) in the order of thousands of milliseconds. For latency-sensitive cloud applications, this may compromise their performance. To overcome this, fog, mist, and edge computing have been introduced as extensions of cloud networks, which are collections of servers comprising a distributed network. Cloud, fog, and mist computing are also known as wet computing in the literature. The difference between them is the degree of distribution of services from the internet cloud to edge devices.

We are introducing a new concept called Flying Edge computing (FEC). In contrast to previous forms of computing, we name it "dry" computing because data are blown along and dried by with the current of the computing wind. The speed and direction of the wind also matter. The faster the speed, the more the throughput. At slower speeds, a network is more tightly squeezed to collect the data.

\section{A. Data evaporation}

UAV activates ground sensor nodes and provides them local computation, database, and communications infrastructure services. In contrast to typical fixed-edge computing machines installed near or inside the edge node, the UAV acts like a flying edge that moves between edge nodes to provide them the desired local and global services. Typical cloud, fog, and mist computing are called wet computing, but for Flying Edge we name it dry, wind, or breeze computing where data are evaporated from the sensor node to the air and collected by a UAV. UAV generates a computing wind in its vicinity, and data are evaporated (extracted from sensors) with the current of computing (movement of UAV). The speed of the UAV is taken as the current of computing wind, while several UAVs act like wind temperature. The more the UAVs, the faster the evaporation process (more throughput). Fast-moving UAVs produce shorter clusters with fewer sensor nodes, so slowing the computing wind ensures that the sensor nodes are squeezed well.

We have taken agriculture in the Kingdom of Saudi Arabia (KSA) as a case study. Crops are mostly grown in circular farms to save water. Our contribution is to modernize the KSA agriculture with state-of-the-art technologies to monitor crop health so resources can be managed tightly, especially the maximal use of water. We map flying edge computing with the perspective of KSA agriculture. 
Hundreds of crop fields are dispersed in a very large and remote area where there is no fixed communications infrastructure. It is assumed that all fields are equipped with a large number of sensors that monitor crops, soil, and environmental parameters at the same time. However, predefined network topologies (cluster heads, communication routes) are not possible because of the high fluctuation of sensor nodes caused by harsh water. In this scenario, cloud computing does not fit well as loT devices do not have cloud access. In this presentation, a flying edge machine (UAV) moves in a predefined path. As it blows the computing wind, the underlying sensors form a cluster in the direction of the wind, and they choose a Cluster Head $(\mathrm{CH})$ dynamically. As a result, the data are evaporated from $\mathrm{CH}$ to UAV, as shown in Figure 1.

\section{B. Comparison of flying edge computing with existing methods}

A comparison of flying edge computing with other technologies, including cloud, fog, mist, and edge computing, is shown in Table 1 and represented in Figure 2.

Table 1: Comparison of different computing techniques

\begin{tabular}{|c|c|c|c|c|c|}
\hline & $\begin{array}{c}\text { Mobile Cloud } \\
\text { Computing (MCC) }\end{array}$ & $\begin{array}{l}\text { Mobile Fog Computing } \\
\text { (MFC) }\end{array}$ & $\begin{array}{c}\text { Mobile Mist } \\
\text { Computing (MMC) }\end{array}$ & $\begin{array}{c}\text { Mobile Edge } \\
\text { Computing (MEC) }\end{array}$ & $\begin{array}{c}\text { Flying Edge } \\
\text { Computing (FEC) }\end{array}$ \\
\hline 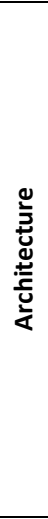 & $\begin{array}{l}\text { - Based on central } \\
\text { processing } \\
\text { - Accessed through the } \\
\text { internet } \\
\text { - Allows a large amount of } \\
\text { data to be accessed more } \\
\text { quickly, this demand is } \\
\text { ever-growing due to } \\
\text { cloud agility }\end{array}$ & $\begin{array}{l}\text { - Coined by cisco } \\
\text { - Extending cloud to the } \\
\text { edge of the networks } \\
\text { - Decentralized computing } \\
\text { - Any device with } \\
\text { computing capability, } \\
\text { storage, and network } \\
\text { connectivity can be a fog } \\
\text { node }\end{array}$ & $\begin{array}{l}\text { - Middle ground } \\
\text { between cloud and Fog } \\
\text { - Lightweight computing } \\
\text { resides in the network } \\
\text { fabric using micro- } \\
\text { controllers and } \\
\text { microchips } \\
\text { - Not a mandatory layer } \\
\text { for fog computing }\end{array}$ & $\begin{array}{l}\text { - Can work without } \\
\text { cloud and fog } \\
\text { - Limited to the } \\
\text { number of peripheral } \\
\text { layers } \\
\text { - Pushes the } \\
\text { intelligence, } \\
\text { processing power, } \\
\text { and communication } \\
\text { of an edge gateway } \\
\text { of appliances directly } \\
\text { into devices like } \\
\text { programmable } \\
\text { automation } \\
\text { controllers (PACs) }\end{array}$ & $\begin{array}{l}\text { - Can work with no } \\
\text { communications } \\
\text { infrastructure } \\
\text { - A mobile node acts } \\
\text { like a commuting } \\
\text { and } \\
\text { communication } \\
\text { machine } \\
\text { - Computing and } \\
\text { communications } \\
\text { node are closer to } \\
\text { the end devices to } \\
\text { provide services }\end{array}$ \\
\hline ŏ & $\begin{array}{l}\text { - Scalable } \\
\text { - Cost-effective } \\
\text { - Based on an internet- } \\
\text { driven global network on } \\
\text { robust TCP/IP protocol }\end{array}$ & $\begin{array}{l}\text { - Real-time data analysis } \\
\text { - Takes quick action } \\
\text { - Sensitive data remain } \\
\text { inside the network } \\
\text { - Saves storage and } \\
\text { network costs } \\
\text { - More scalable than mist } \\
\text { and edge } \\
\text { - Operations can be } \\
\text { managed by the IT team } \\
\text { - Fog node can be } \\
\text { deployed in protected, } \\
\text { private, public, or hybrid } \\
\text { mode }\end{array}$ & $\begin{array}{l}\text { - Local decision-making } \\
\text { data } \\
\text { - Works with fog } \\
\text { computing and cloud } \\
\text { computing }\end{array}$ & $\begin{array}{l}\text { - More secure } \\
\text { - Fast } \\
\text { - Not dependent on } \\
\text { the internet }\end{array}$ & $\begin{array}{l}\text { - Completely secure } \\
\text { - No need for the } \\
\text { internet or any } \\
\text { communications } \\
\text { infrastructure } \\
\text { - Saves power } \\
\text { - Scalable } \\
\text { - Works well under } \\
\text { hard conditions } \\
\text { and where sensing } \\
\text { devices are } \\
\text { fluctuating rapidly }\end{array}$ \\
\hline 气ุ & $\begin{array}{l}\text { - Long latency/response } \\
\text { time } \\
\text { - limited bandwidth } \\
\text { - Security vulnerabilities } \\
\text { - No offline mode } \\
\text { - Bottlenecks if many } \\
\text { devices send data } \\
\text { simultaneously }\end{array}$ & $\begin{array}{l}\text { - Data must pass through } \\
\text { many links to approach } \\
\text { the fog node } \\
\text { - Single point of failure }\end{array}$ & & $\begin{array}{l}\text { - Less scalable } \\
\text { - Requires more node } \\
\text { energy } \\
\text { - No cloud awareness } \\
\text { - Not usable in hard } \\
\text { conditions or where } \\
\text { sensor nodes are } \\
\text { fluctuating }\end{array}$ & \\
\hline
\end{tabular}




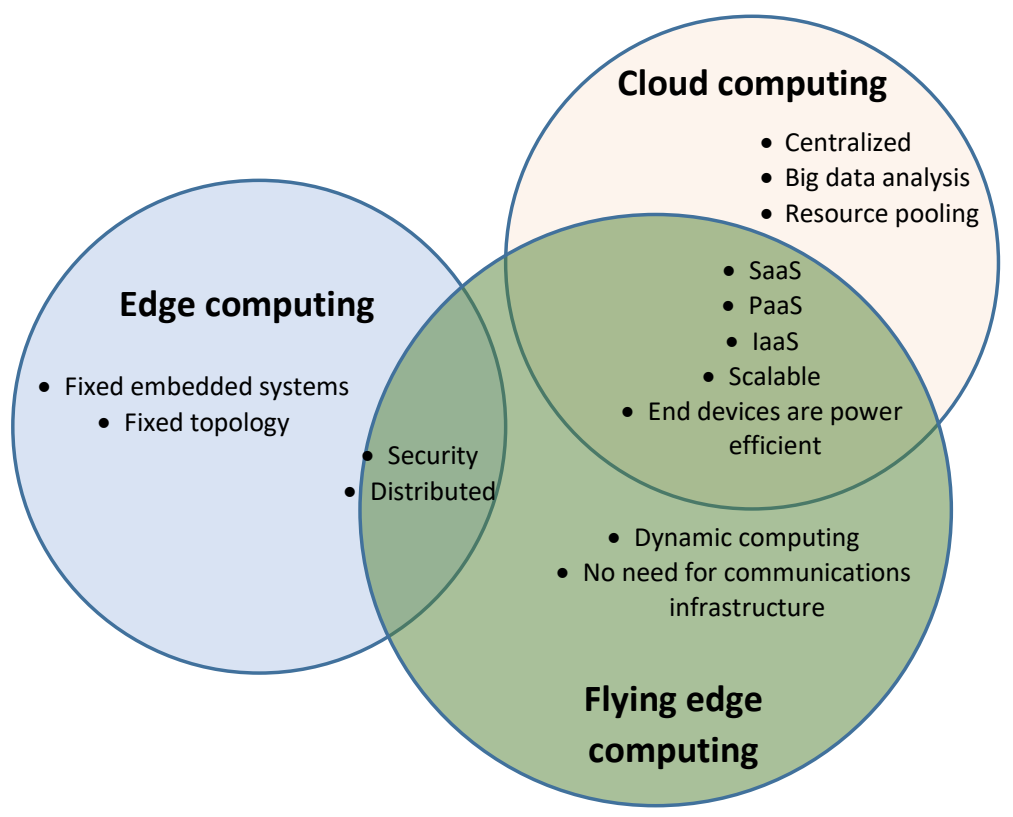

Figure 2 : Similarities and differences of computing techniques

As mentioned above, the Flying Edge is a UAV-based platform that acts as an edge node. Most importantly, the acting edge node can be reallocated to different locations where various sensors or devices need some edge node to complete certain loT tasks. Moreover, it can provide the facility of a gateway to link or integrate loT services and cloud computing. The Flying Edge can be equipped with resources like processors, storage facilities, communication devices, and an edge computational platform. This edge platform can execute several services to support loT applications required for smart agriculture. One possible configuration for flying edge computing is shown in Figure 3 . In that figure, a UAV with edge computing facilities can move in any area where loT services are required. It provides a set of services like low latency to the loT applications in that area while it enables the integration of the loT applications and the cloud.
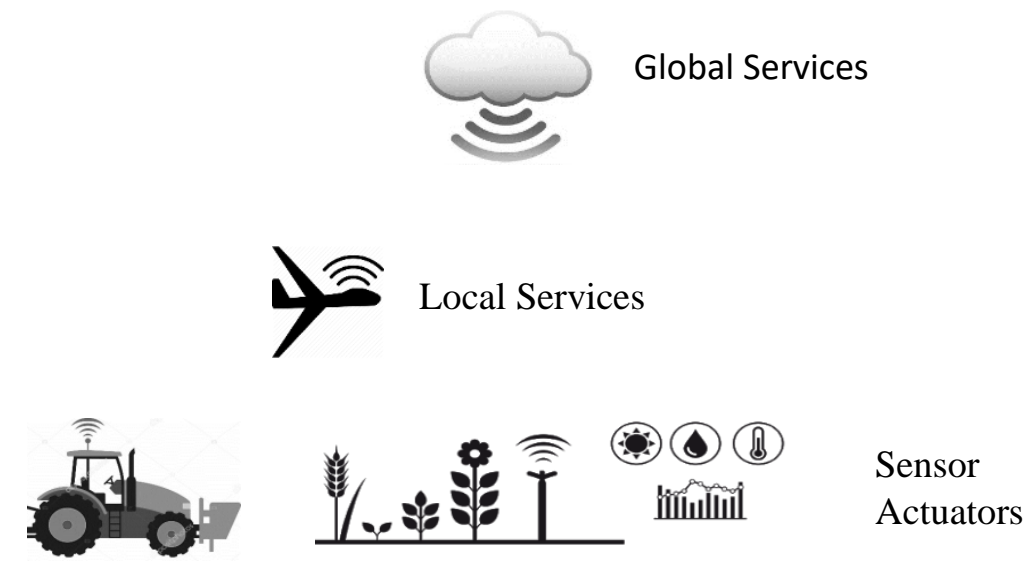

Figure 3: Flying Edge supporting the smart agriculture

The Flying Edge can facilitate smart agriculture based on loT applications. Major opportunities that it can provide are: 
- Fast deployment: because of its nature, the Flying Edge can be deployed at remote locations to facilitate loT services. It is a turnkey system that can be used even where there is no communications infrastructure.

- Scalability: the system can be upgraded or enhanced in terms of coverage area, number of sensing devices, number of UAVs operated, and services provided.

- Flexibility: the system is flexible enough to adapt to any change in applications, required services, or the geography of the monitoring area.

- Location awareness: location services are provided by the flying edge machine, so loT nodes do not require a GPS device.

- Power effectiveness: this scheme is very power efficient for end nodes (IoT nodes), as most of the services that require computation are provided by flying edge robots (UAVs).

Because the Flying Edge can be deployed in an area where loT devices are already deployed, most of the communication between these loT devices and UAV is done through a personal area network (PAN) such as IEEE 802.15.4 (Zigbee) and 801.15.1 (Bluetooth). Both protocols are characterized by lower bandwidths and shorter communication ranges. However, they also need low energy during the communication phase. Depending on the requirements, communication can be done through alternative protocols that support longer ranges like a local area network (LAN) such as IEEE 802.11 (Wi-Fi). On the other hand, all the communication between the Flying Edge and the cloud is done through protocols that are commonly used for wide area networks (WANs) including IEEE 802.16 (WiMAX) and cellular. The choice of communications medium and protocol depends on the nature of the application and its location.

\section{Proposed system architecture}

The proposed system has two components: an edge device and the Flying Edge computing machine, as shown in Figure 4. The edge device (sensor or actuator) can get services from a UAV through a services request interface. Most of the computational services required by the loT are provided by the local service response module, which is connected to several services built into the flying machine. In rare cases, the loTs might require global services from the cloud, and they will be granted through a global services response module.

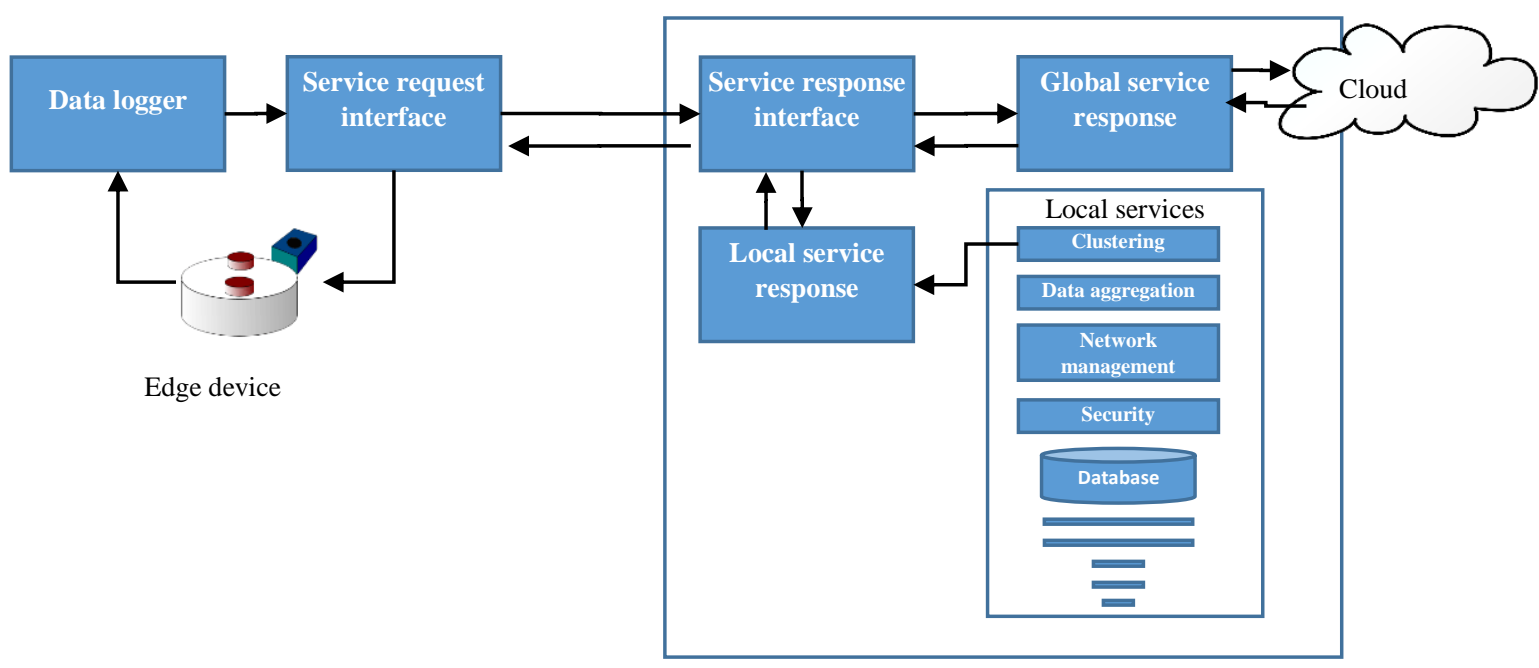

Flying Computing Machine 


\section{Proposed system models}

Mobile devices and Internet-of-things (IOT) have been introduced in our lives very rapidly. They have brought ease to our work, but they also raised new challenges that needed cloud-based solutions including i) the need to transfer huge amounts of data to the cloud, ii) high communication latencies, and iii) the inability of this model to respond in some domains that require a rapid reaction to events. Different computing techniques like cloud, fog, and mist/edge computing have been introduced to address these issues and provide three basic services to the loT devices: Infrastructure as a Service (laaS), "Platform as a Service (PaaS), and Software as a Service (SaaS). The basic difference between these techniques is the layer in the network hierarchy from which the service is provided. This means the distance between the services and the source of data, as shown in Figure 5. In cloud computing, all the services are provided from the cloud (a central location) while fog computing is a distributed computing technique in which services are provided in network fabrics. In edge or mist computing, processing and services are provided near the end node (the source of data).

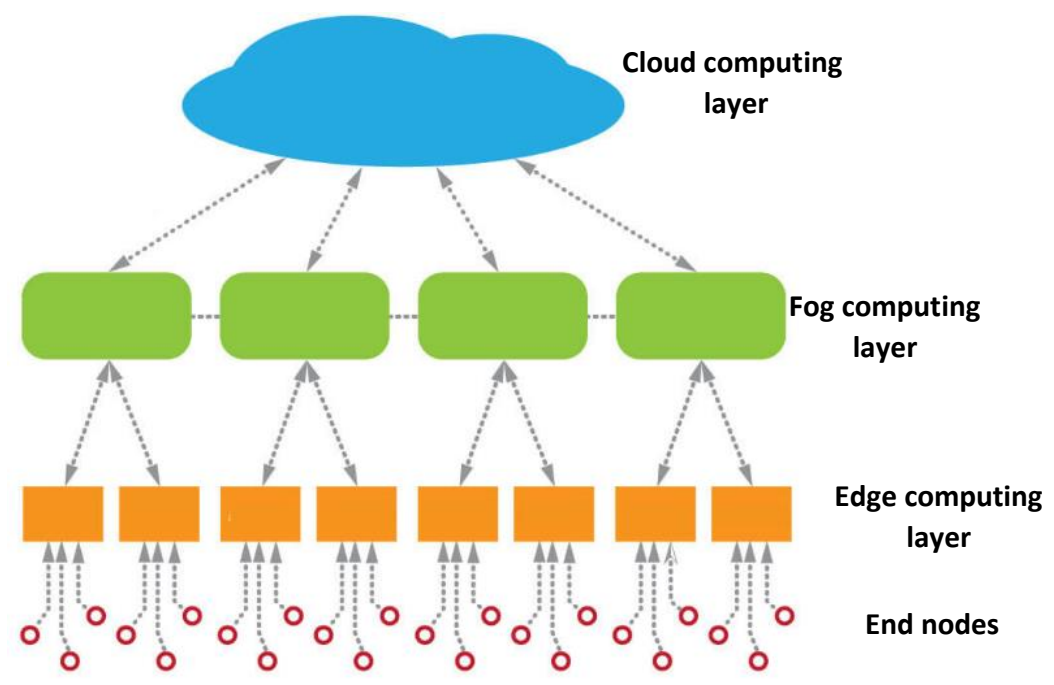

Figure 5: Typical cloud-to-edge computing hierarchy

A typical computing hierarchy is shown in Figure 5. As far as WSN is involved, many factors (lack of a communication link, limited processing capabilities, insufficient power) may restrict the use of any kind of computing technique. In such conditions, a UAV can replace any of the computing layers. In this article, we introduce four basic types of UAV replacement:

1- UAV as a flying cloud

2- UAV as a flying fog

3- UAV as a flying edge

4- A swarm of UAVs as a distributed services provider

\section{1) UAV as a flying cloud}

This configuration is applicable in a scenario where all the ground sensing nodes can communicate with each other but do not have access to the outer world (no internet). This type of scenario is applicable in battlefields, disaster areas, wildlife habitats, and remote farm fields. As all sensors are connected with each other, they maintain their local network in terms of clusters and routes independently. Local decisions are made either at the edge or the fog layer. Global services (data collection, localization, security, or software) and decisions are provided by the UAV as a Flying Cloud Machine (FCM) on a demand basis (see Figure 6). 

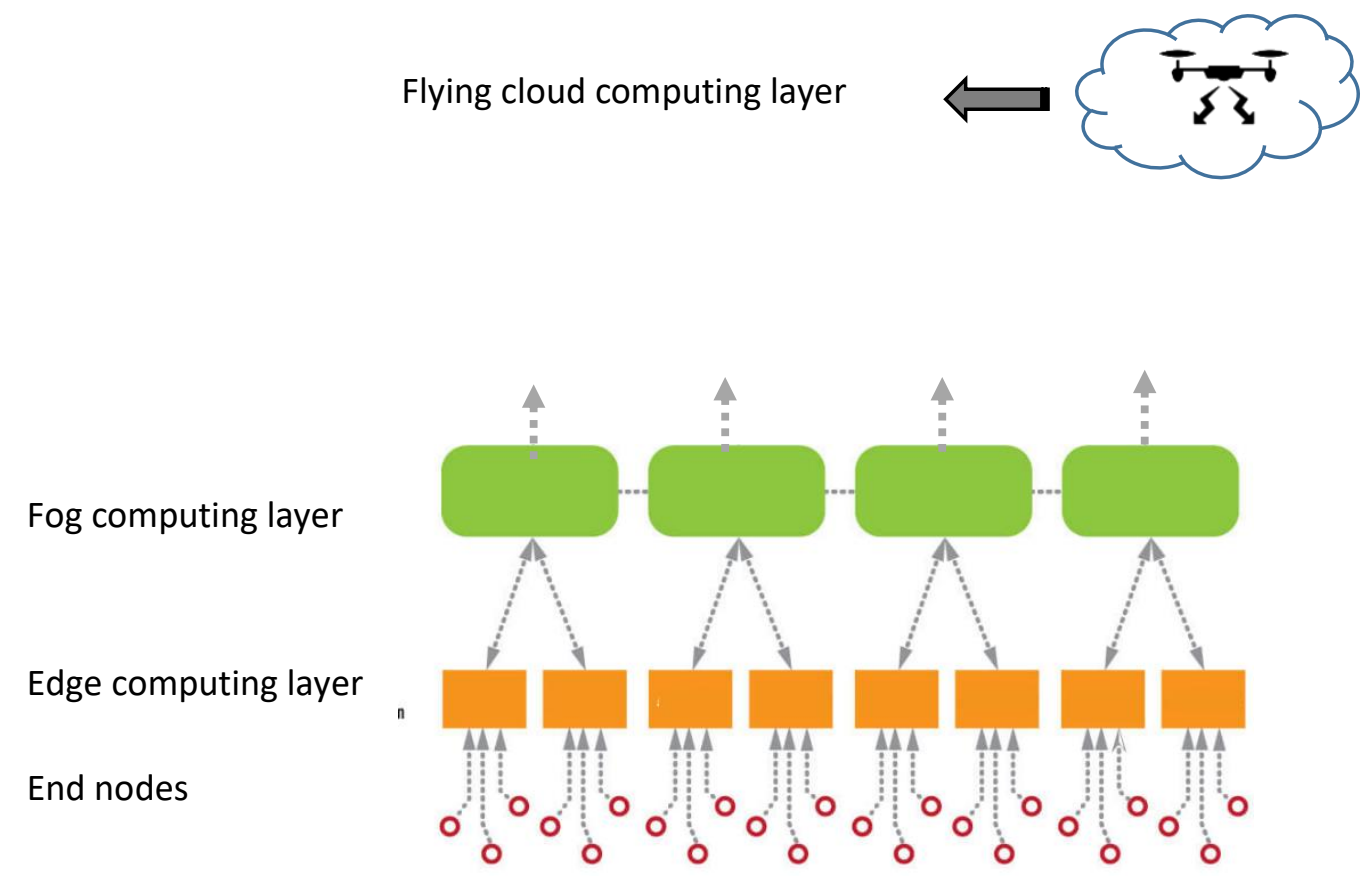

Figure 6: UAV a Flying cloud machine

\section{2) UAV as a flying fog}

In this configuration, field sensors are equipped with local services (network and storage) through edge nodes. The UAV provides only communication services as a flying fog machine (FFM) to approach the cloud layer where all global services exist. FFM provides a bridge between the edge layer and the cloud layer, as shown in Figure 7.

\section{Cloud computing} machine

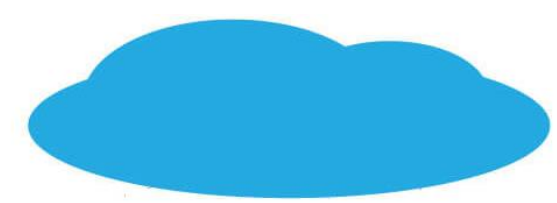

Flying fog computing machine
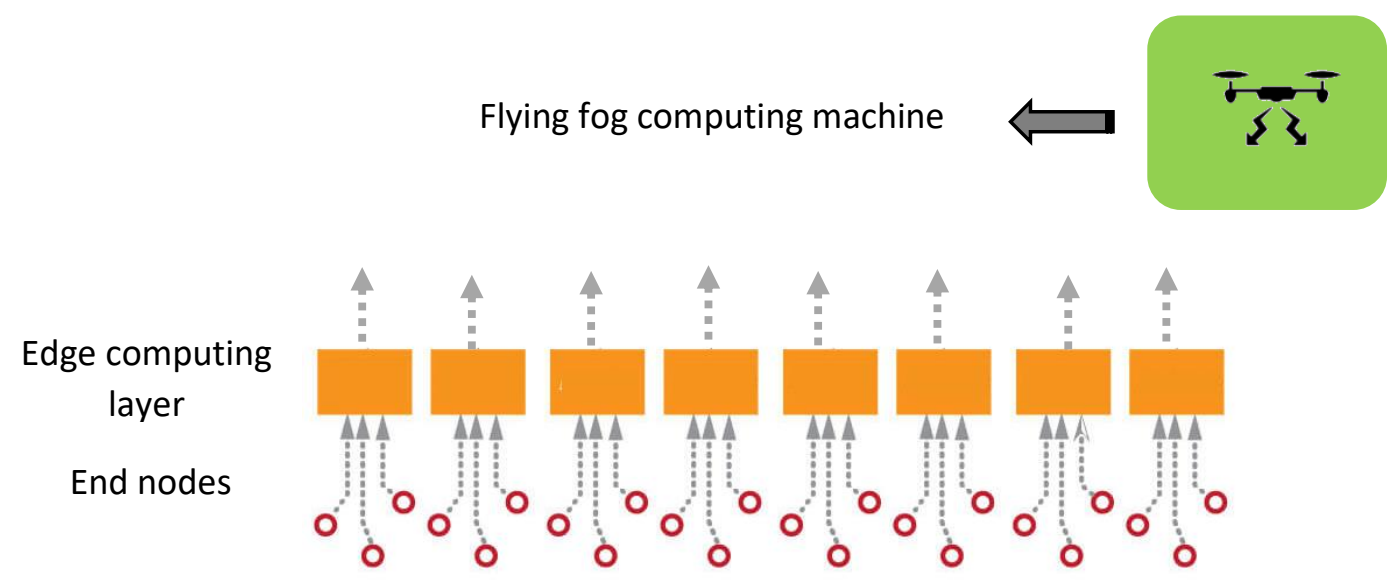

Figure 7: Flying fog computing 


\section{3) UAV as a flying edge}

In this scheme, UAV provides computation and services to the loT nodes by visiting them, and it provides services and computation close to the data source. It helps to improve latency and bandwidth, and it also enhances the network life by improving battery utilization of end nodes. All the sensors' nodes are dump nodes. Their functionality is limited to sense and log parameters from the environment only. All the services (clustering, network, storage) and computation are provided to them by a UAV. If the UAV cannot fulfill a demanded service, it can render it from the cloud. A flying edge machine (FEM) is shown in Figure 8.

Cloud computing layer

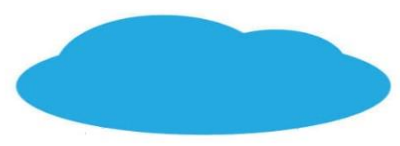

Flying edge computing machine

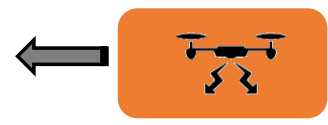

IoT

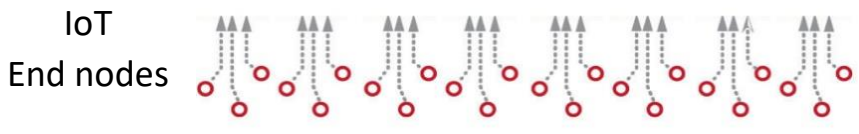

Figure 8: Flying edge computing

\section{4) UAV as a distributed flying edge machine}

A coordinated swarm of UAVs can provide edge services as shown in Figure 9. Different services like network, storage, and localization are provided by separate UAVs. Hence each service will be provide by a specialized flying machine which will enhance the provided quality of service, throughput, and data collection. Swarm of UAVs can also work in a coordinated way such that they can share or take over the responsibilities if any one fails, distracted or power exhausted. This model will provide robust solutions in hardship conditions.

Cloud computing layer
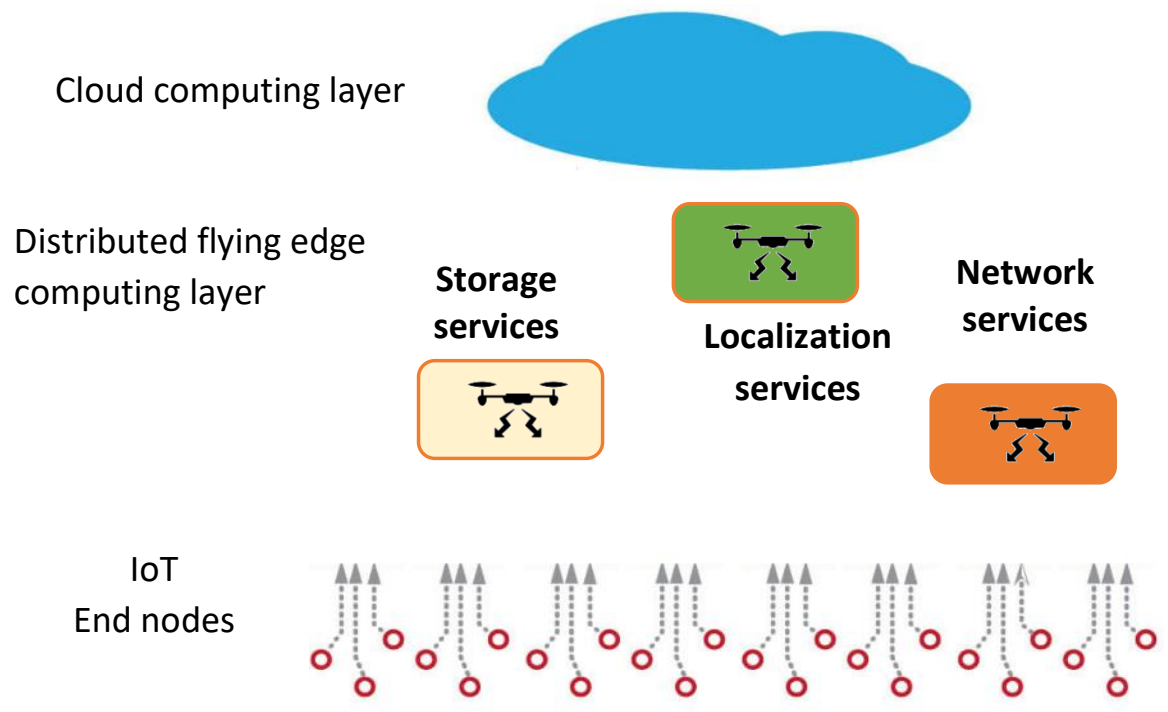


\section{Simulation and Results}

A Flying Edge prototype was implemented using a distributed Java agent environment. This environment provides a middleware infrastructure that enables the implementation of different distributed programming models and advanced platform services for distributed heterogeneous environments. These heterogeneous environments may comprise multiple heterogeneous nodes with different capabilities. In addition, this middleware infrastructure provides scalability solutions to deploy, operate, monitor, and control large-scale distributed applications. Each agent in the environment can provide runtime support to securely execute some code. An edge machine provides broker services, invocation services, and location-based services. There is also a mechanism that allows each edge broker to forward a service search request to other brokers if it does not have the requested service. Both local and remote service invocations also are implemented and added to the prototype implementation.

For the loT side, we used the Arduino board, which is open-source hardware for embedded systems. For this prototype implementation, the Arduino was used as the loT payload subsystem-the onboard device requesting services. Some sensors were connected to the Arduino board, such as the DHT11 sensor to measure temperature and humidity. Some LEDs and a buzz were installed to represent actuators. In addition, we installed an Adafruit CC3000 Wi-Fi board to connect the Arduino to a local area network and the Flying Edge machine. The Arduino code was developed using the Arduino IDE with the Adafruit CC3000 library. Each loT service was implemented with a RESTful API.

At the UAV edge computing side, there is a service that represents each sensor or actuator attached to the Arduino. The primary function of these services is to map a call from the SOAP APIs to RESTful APIs. All sensor and actuator services are registered with the local broker. In addition, the global broker is periodically updated with these services. For the experiments, we used three computers, which represented the cloud, the Flying Edge machine, and the loT node. In addition, we used WAN emulators among these machines to introduce the effects of using long distances and/or the internet to connect the machines. Experiments were conducted for four configurations:

- Call1: a local loT service call responded by the distributed flying edge machine

- Call2: a local loT service call responded by the flying edge machine

- Call3: a remote loT service call responded by the cloud through a flying fog machine

- Call4: a remote loT service call responded by the flying cloud

The experiment was repeated for two types of services. The first was to get the current temperature (CurTemp) and the second was to turn on the LED (LEDon). The average results of 10 runs of multiple service calls are shown in Figure 10. The recorded times for these calls do not include the service lookup times. The response times for all service calls are shown in Figure 10. It is evident that flying edge shows best results in term of response time by adding the benefits of battery efficiency of end nodes. It is also analyzed that the services provided by flying fog machine or through flying fog machine gives same results and are better than cloud. 


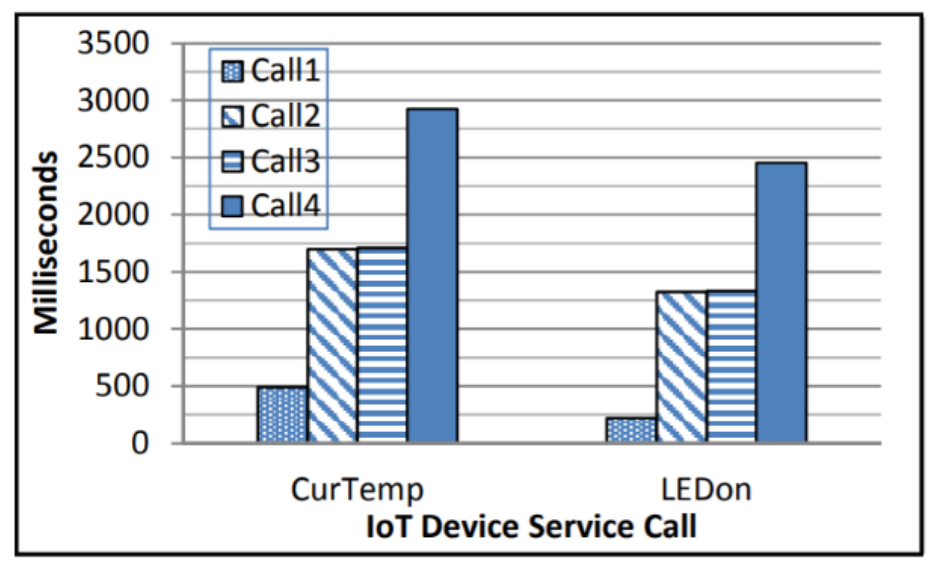

Figure 10: IoT service call response time

The average service lookup times for three services are shown in Figure 11. Local services were provided by the local network. Remote 1 services were provided by the flying machine UAV to the end node. Remote 2 services were provided by the cloud through flying machines. There is a big difference between Remote 1 and Remote 2 service lookup times. This means that flying computing machines (UAVs) can provide services to loT devices in a much faster way.

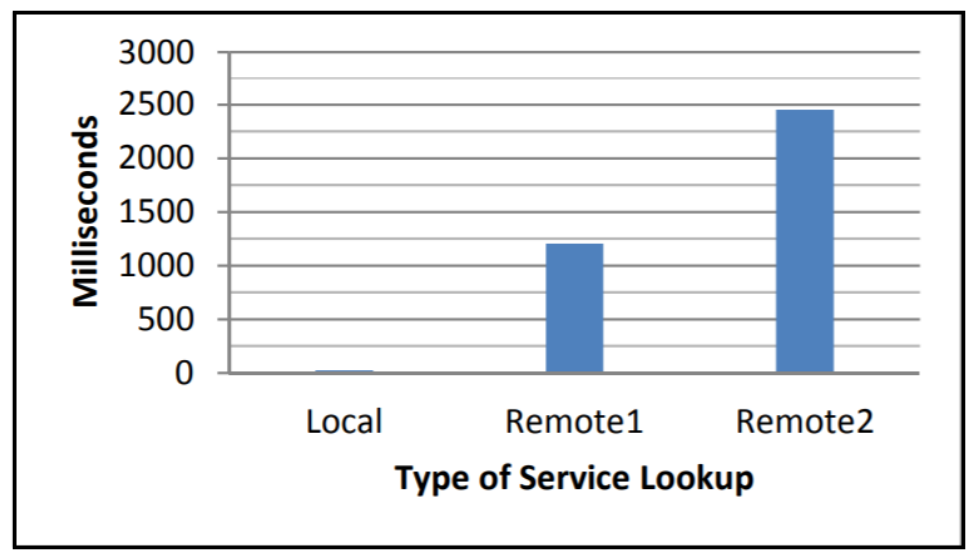

Figure 11: IoT service lookup

\section{Conclusion}

In this research article, we proposed a new concept of a computation mechanism for loT devices. The processing and the services will be provided to the end devices neither from central location (cloud) nor by end device itself. Both cloud and edge computing have different pros and cons ranging from bottlenecks to poor resource utilization. The proposed system fulfills the service demand very close to the point of data generation without using additional network resources (energy, memory, or processor). Our aim is to provide these services to the loTs by using the Flying Edge machine. This means the computational machine itself goes closer to the data-generation node and provides the demanded services. The architecture of the proposed Flying Edge machine is introduced, and the performance of the system is evaluated through simulation and lab test bed. The results showed the effectiveness of the proposed computational mechanism. As a result, we can say this system is very suitable for implementation in the case study presented (agriculture) but it is not limited to that use. 


\section{Acknowledgement}

The authors gratefully acknowledge the support of SNCS Research Center at the University of Tabuk, Saudi Arabia.

\section{References}

[1] O. Friha, M. A. Ferrag, L. Shu, L. Maglaras, and X. Wang, "Internet of Things for the Future of Smart Agriculture: A Comprehensive Survey of Emerging Technologies," IEEE/CAA J. Autom. Sin., vol. 8, no. 4, pp. 718-752, Apr. 2021.

[2] M. Ammad Uddin, M. Ayaz, E.-H. M. Aggoune, A. Mansour, and D. Le Jeune, "Affordable broad agile farming system for rural and remote area," IEEE Access, vol. 7, 2019.

[3] A. Mishra, N. Gupta, and B. B. Gupta, "Defense mechanisms against DDoS attack based on entropy in SDN-cloud using POX controller," Telecommun. Syst., Jan. 2021.

[4] C. Yu, J. Li, X. Li, X. Ren, and B. B. Gupta, "Four-image encryption scheme based on quaternion Fresnel transform, chaos and computer generated hologram," Multimed. Tools Appl., vol. 77, no. 4, pp. 4585-4608, Feb. 2018.

[5] P. K. R. Maddikunta et al., "Unmanned Aerial Vehicles in Smart Agriculture: Applications, Requirements, and Challenges," IEEE Sens. J., pp. 1-1, 2021.

[6] K. Ivushkin et al., "UAV based soil salinity assessment of cropland," Geoderma, vol. 338, pp. 502-512, Mar. 2019.

[7] S. A. Suab and R. Avtar, "Unmanned Aerial Vehicle System (UAVS) Applications in Forestry and Plantation Operations: Experiences in Sabah and Sarawak, Malaysian Borneo," in Unmanned Aerial Vehicle: Applications in Agriculture and Environment, Cham: Springer International Publishing, 2020, pp. 101-118.

[8] J. Valente, B. Sari, L. Kooistra, H. Kramer, and S. Mücher, "Automated crop plant counting from very high-resolution aerial imagery," Precis. Agric., vol. 21, no. 6, pp. 1366-1384, Dec. 2020.

[9] M. A. Uddin, A. Mansour, D. Le Jeune, M. Ayaz, and el-H. M. Aggoune, "UAV-Assisted Dynamic Clustering of Wireless Sensor Networks for Crop Health Monitoring," Sensors, vol. 18, no. 2, p. 555, Feb. 2018.

[10] M. Ammad-Uddin, A. Mansour, D. Le Jeune, E. H. M. Aggoune, and M. Ayaz, "UAV Routing Protocol for Crop Health Management," in 24th European Signal Processing Conference (EUSIPCO), 2016, pp. 1818-1822.

[11] E.-H. Aggoune, S. Alwakeel, M. Alwakeel, and M. Ammad-Uddin, "Wireless Sensor Networks with Dynamic Nodes for Water and Crop Health Management," in Sensor Networks for Sustainable Development, CRC Press, 2014, pp. 27-40.

[12] A. M. El-Shamy, N. A. El-Fishawy, G. Attiya, and M. A. A. Mohamed, "Anomaly Detection and Bottleneck Identification of The Distributed Application in Cloud Data Center using SoftwareDefined Networking," Egypt. Informatics J., Jan. 2021.

[13] K. Hong, D. Lillethun, U. Ramachandran, B. Ottenwälder, and B. Koldehofe, "Mobile fog," in Proceedings of the second ACM SIGCOMM workshop on Mobile cloud computing - MCC'13, 2013, p. 15.

[14] C. L. Stergiou, K. E. Psannis, and B. B. Gupta, "IoT-based Big Data secure management in the Fog over a 6G Wireless Network," IEEE Internet Things J., pp. 1-1, 2020.

[15] H. T. Dinh, C. Lee, D. Niyato, and P. Wang, "A survey of mobile cloud computing: architecture, 
applications, and approaches," Wirel. Commun. Mob. Comput., vol. 13, no. 18, pp. 15871611, Dec. 2013.

[16] A. Ahmed and E. Ahmed, "A survey on mobile edge computing," in 2016 10th International Conference on Intelligent Systems and Control (ISCO), 2016, pp. 1-8.

[17] C. Esposito, M. Ficco, and B. B. Gupta, "Blockchain-based authentication and authorization for smart city applications," Inf. Process. Manag., vol. 58, no. 2, p. 102468, Mar. 2021.

[18] X. Chen, Q. Shi, L. Yang, and J. Xu, "ThriftyEdge: Resource-Efficient Edge Computing for Intelligent loT Applications," IEEE Netw., vol. 32, no. 1, pp. 61-65, Jan. 2018.

[19] G. Milics, "Application of UAVs in Precision Agriculture," in International Climate Protection, Cham: Springer International Publishing, 2019, pp. 93-97.

[20] P. Sethi and S. R. Sarangi, "Internet of Things: Architectures, Protocols, and Applications," J. Electr. Comput. Eng., vol. 2017, pp. 1-25, 2017.

[21] X. Li, Z. Ma, J. Zheng, Y. Liu, L. Zhu, and N. Zhou, "An Effective Edge-Assisted Data Collection Approach for Critical Events in the SDWSN-Based Agricultural Internet of Things," Electronics, vol. 9, no. 6, p. 907, May 2020.

[22] M. A. Zamora-Izquierdo, J. Santa, J. A. Martínez, V. Martínez, and A. F. Skarmeta, "Smart farming loT platform based on edge and cloud computing," Biosyst. Eng., vol. 177, pp. 4-17, Jan. 2019. 